\title{
Entretien d'Alain Mahe : Itinéraire d'une recherche. L'engagement civique d'un anthropologue en Kabylie
}

Interview with Alain MAHE: Itinerary of a Research: The civic engagement of an anthropologist in Kabylie

\section{Daniel Cefaï}

\section{(2) OpenEdition Journals}

\section{Édition électronique}

URL : http://journals.openedition.org/conflits/844

DOI : $10.4000 /$ conflits.844

ISSN : $1777-5345$

\section{Éditeur :}

CCLS - Centre d'études sur les conflits lilberté et sécurité, L'Harmattan

\section{Édition imprimée}

Date de publication : 1 septembre 2002

Pagination : 171-192

ISBN : 2-7475-3432-4

ISSN : 1157-996X

\section{Référence électronique}

Daniel Cefaï, «Entretien d'Alain Mahe : Itinéraire d'une recherche. L'engagement civique d'un anthropologue en Kabylie », Cultures \& Conflits [En ligne], 47 | automne 2002, mis en ligne le 29 avril 2003, consulté le 30 mars 2021. URL : http://journals.openedition.org/conflits/844 ; DOI : https:// doi.org/10.4000/conflits.844

Ce document a été généré automatiquement le 30 mars 2021.

Creative Commons License 


\title{
Entretien d'Alain Mahe : Itinéraire d'une recherche. L'engagement civique d'un anthropologue en Kabylie
}

\author{
Interview with Alain MAHE: Itinerary of a Research: The civic engagement of an \\ anthropologist in Kabylie
}

Daniel Cefaï

Daniel Cefaï : Commençons par le commencement. D'où te vient ta passion pour ton objet d'enquête ? A qui te connait, il est évident qu'elle déborde de beaucoup le simple engagement intellectuel. Peux-tu décrire l'enracinement biographique de cette passion?

Alain Mahé : Quand on me pose cette question, j'ai l'habitude de donner la réponse d'Obélix : la Kabylie, je suis tombé dedans quand j'étais petit. Plus sérieusement, j'ai vécu toute mon enfance et mon adolescence dans une cité de la banlieue sud de Paris, à Créteil, où il y avait beaucoup d'enfants issus de l'immigration maghrébine. Nombre d'entre eux étaient kabyles. Par le plus grand des hasards, il se trouve que mon ami d'enfance - comme un frère-, a très tôt été sensibilisé par la question berbère en Algérie. Dès le début des années 1970. A ce moment-là, il faut savoir que c'était en France que se trouvaient les principaux relais de ce qui deviendra dans les années 1980 le Mouvement culturel berbère (MCB). C'est ici, en immigration, qu'avaient lieu les manifestations et qu'opérait la coopérative d'édition et de diffusion berbère -Imdiazen- qui distribuait la littérature militante, les brochures et les tracts. Comme je partageais presque tout avec cet ami, je l'ai suivi. C'est ainsi qu'en sa compagnie j'ai commencé à apprendre le Kabyle en traduisant mes leçons d'espagnol en berbère. Je venais d'entrer en classe de 4ème, j'avais donc 12-13 ans - j'ai toujours ce cahier de kabyle/espagnol. Quelques années plus tard, je suis allé suivre quelques cours de berbère à Censier, et encore un peu plus tard, j'ai même étudié trois ou quatre poèmes - de Ait Menguellet et de Idir - pour préparer le kabyle en tant qu'épreuve facultative au bac. Je ne sais pas pourquoi, je n'ai pas été convoqué alors que mes copains l'ont été! Cela dit je bafouillais à peine le kabyle, et j'avais fait cela par défi. 
Hormis cet aspect affectif, mon rapport à la Kabylie a été d'emblée politique. J'ai fait ma première manif' devant l'ambassade d'Algérie en 1976. Je me souviens, on était deux ou trois cents et je garde encore en mémoire le visage d'un homme plus âgé que la moyenne des manifestants, que les organisateurs de la manif nous avaient désigné comme un provocateur, en nous mettant en garde, nous les jeunes (j'avais 16 ans). C'est en 1978, dès que j'ai atteint la majorité légale, que je suis allé pour la première fois en Kabylie. J'avais tout simplement fait partie du voyage familial qui amenait périodiquement les parents de mon copainaubledpour des vacances d'été. Depuis lors et jusqu'en 1992 j'y ai fait au moins un long séjour par an.

En avril 1980 explose en Kabylie le premier mouvement social de l'Algérie indépendante, d'une amplitude qui étonne jusqu'à ses initiateurs, qui avaient appelé à manifester dans le milieu scolaire, universitaire et syndical. C'est juste à cette époque que je venais de rejoindre le département de sociologie de Nanterre, en vue de préparer par la suite une licence d'anthropologie. J'avais alors plutôt en tête d'étudier un sujet lié à l'Afrique noire, j'ai longtemps côtoyé des Sénégalais et des Mauritaniens et pas mal voyagé dans ces régions. Les développements du mouvement culturel berbère ont été décisifs pour me réorienter vers le Maghreb. D'autant que ce qui caractérise la décennie de lutte des années 1980, c'est une assez forte mobilisation dans l'immigration et une large couverture médiatique. J'ai donc très vite été happé par ce mouvement et c'est ainsi qu'à Paris X, sous la direction de Gianni Albergoni, j'ai choisi un sujet de maitrise sur l'histoire des luttes symboliques autour de l'identité kabyle. A ce moment-là, pour moi, l'enjeu n'était pas tant d'étudier la Kabylie que d'analyser les discours qu'on tenait sur elle. En effet, avec la lecture de la littérature ethnographique sur la région et celle de la presse française, je commençais à entrevoir dans les discours que les Kabyles tenaient sur leur région et sur leur propre identité des propos qui m'intriguaient. Je me proposais donc de passer tous ces discours au crible d'une sociologie de la connaissance. Le but étant de périodiser les modèles d'explication, les clichés et les stéréotypes proposés sur la Kabylie. Très vite je m'étais aperçu que ce qu'on appelle le «mythe kabyle» des Français excédait de beaucoup la dimension idéologique sur laquelle les analystes s'étaient polarisés. De surcroît, à la faveur de la réappropriation par les Kabyles du savoir produit sur eux durant la période coloniale - réappropriation dont procède largement le $\mathrm{MCB}$ - le mythe et ses clichés étaient devenus un élément du discours identitaire des Kabyles sur eux-mêmes.

C'est ainsi que j'ai mis en perspective les divers types de savoir produits sur la Kabylie depuis l'ethnographie des officiers des affaires indigènes jusqu'à la littérature universitaire et militante des Kabyles contemporains. C'est également dans cette perspective que j'ai été amené à m'intéresser à la chanson kabyle qui a été durant toute la décennie 1980 le principal vecteur de la revendication culturelle. Avec la libéralisation de la presse depuis 1990, la situation a notablement changé sous cet aspect. Dans mon DEA, j'ai même présenté une discographie commentée des principaux compositeurs associés au mouvement culturel - Ait Menguellet, Ferhat (du groupeImazighen Imoula), Djemal Alam, Idir et, évidemment, Matoub Lounès.

Paradoxalement, ma maîtrise de la langue kabyle n'a jamais été bonne. Et ce n'est pas le « dulco » de Berbère que j'ai partiellement suivi à l'Inalco qui a amélioré ma pratique. En fait, je baragouinais suffisamment pour donner le change dans des situations quotidiennes. Ma compétence linguistique était tout à fait comparable à celle de bien des jeunes de ma génération issus de l'immigration kabyle en France. Ma première 
épouse, kabyle, qui comprenait parfaitement la langue de ses parents, ne savait pas la parler et c'est avec moi qu'elle a entrepris d'apprendre à prononcer les phonèmes les plus difficiles. Si je ne m'y suis jamais mis à fond, comme j'aurais dû, c'est qu'en Kabylie, et jusque dans les affaires les plus « traditionnelles » sur lesquelles j'ai travaillé dans le cadre de ma thèse, dès qu'une discussion prend un tour politique ou technique, les gens se mettent à parler en français. Pour te dire, dans la plupart des villages de grande Kabylie, les procès-verbaux des assemblées villageoises sont rédigés en Français, parfois depuis le début du XXe siècle. Ce qui est sûr c'est que dans les villages où les gens m'identifiaient comme Français ce que je savais de kabyle m'a permis de gagner une forme de reconnaissance et de crédit de la part de mes interlocuteurs, sensibles à cet effort de parler leur langue. Pour le reste, dans les villes et bourgades de Kabylie, je passais sans problème pour un Kabyle et j'ai dû, plus d'une fois exhiber ma carte d'identité pour prouver que j'étais Français « de souche ».

Quel est le statut de ce mythe kabyle, dont tu as dû toi-même te départir? De cette chasse aux illusions qui ont tellement pris corps qu'elles sont devenues les évidences de tout le monde?

La critique raisonnée du mythe kabyle avait pour moi une portée politique. La sociologie de la connaissance servait à critiquer les lieux communs de la couverture médiatique des événements, qui reprenaient allègrement les clichés essentialistes sur les Berbères et le thème de l'esprit d'indépendance des Kabyles, ce peuple rude et montagnard, héritier du royaume de Jugurtha et Massinissa. Je voulais montrer que la mobilisation des années 1980 ne devait rien à une identité kabyle multi-séculaire. Je voulais rendre compte de l'atypicité de la Kabylie en recourant à une explication par des conditions socio-historiques qui relèvent pour une part des types d'économie traditionnelle en place avant la colonisation et pour une autre résultent des développements de ces cent cinquante dernières années: les modalités particulières de la conquête et de l'administration de la Kabylie, les divers régimes d'exception d'une "politique kabyle » qui n'a pas toujours dit son nom, mais qui était effective. La politique scolaire est la plus connue, mais il faut aussi mentionner des régimes d'exception en matière de fiscalité, d'administration de la justice (des justices de paix s'appuyaient sur les coutumes kabyles alors que le reste du pays était régi par le droit musulman), ainsi que de nombreuses autres mesures en matière de représentation politique à l'échelle locale. Ce que j'ai voulu montrer c'est que les effets de ces politiques ont été à la fois réels et symboliques. Réels : la scolarisation a par exemple produit une structuration sociale très différente de celle du reste du pays en favorisant l'émergence d'une élite intellectuelle très importante; symboliques: les Kabyles sont passés pour les enfants chéris de la colonisation et se sont eux-mêmes perçus comme porteurs d'un destin spécifique. Très vite, cette région a concentré toutes les contradictions propres à l'Algérie, aggravées par la densité de population et la proximité d'Alger. Et cela est aussi vrai pour la période coloniale, pour rendre compte de la part prise par les Kabyles dans le mouvement nationaliste, que pour l'Algérie indépendante.

Ainsi, dans les années 1970, la répression de toute manifestation de culture berbère n'a été que la goutte d'eau qui a fait déborder le vase. Un élément fondamental pour comprendre la situation de la Kabylie est la sur-politisation et la sur-syndicalisation des Kabyles. La région a donné les premiers et plus importants contingents de l'immigration ouvrière en France. Ce qui leur a assuré ensuite une place de premier rang dans les mouvements politiques et syndicaux algériens. La maîtrise du français par les Kabyles leur a permis d'avoir une connaissance intime de la grammaire de la contestation en France et de la transposer dans les luttes politiques au pays. Un des 
fondateurs d'une des coordinations du mouvement citoyen de ses derniers mois - Amar Fali - que j'interrogeais à ce sujet a eu cette formule formidable : « En Kabylie, la langue française, c'est notre butin de guerre ». Il est frappant que les jeunes qui n'ont pas été scolarisés en français, et qui donc, théoriquement, étaient destinés par un effet de génération à oublier cette langue, continuent à la maîtriser dans les réunions et les manifestations de ces derniers mois. Le français reste la langue politique par excellence. Cette compétence linguistique se transmet dans la famille et au village, au sein des phratries et entre générations, comme le médium des discussions, des critiques et des revendications. Elle se transmet aussi grâce à la télévision, les chaînes officielles étant boudées par les citoyens qui lui préfèrent les programmes transmis via la parabole.

Est-ce que tu peux préciser les méthodes d'enquête que tu as adoptées, et spécifier en particulier la forme qu'y a prise l'articulation entre anthropologie et histoire? Quel y a été le statut de l'analyse cartographique? Fonde-t-elle une sorte de géographie ou d'écologie politique?

Pendant des années, j'ai fait un travail de rat de bibliothèque. A la Bibliothèque Nationale, aux archives du Centre des Hautes Etudes d'Administration Musulmane (CHEAM), dans les universités, à lire les dizaines de thèses inédites, de mémoires de maîtrise et de DEA consacrés à la Kabylie, le plus souvent par des étudiants kabyles. Mon travail a commencé par une critique des sources, une compilation critique des données. Ensuite, j'ai procédé à une exploration de documents de première main sur les rares aspects pas encore étudiés (tels que les centres municipaux). Parallèlement, j'ai longtemps travaillé sur les hypothèses théoriques formulées sur la Kabylie et de façon systématique sur les modélisations anthropologiques des sociétés du Maghreb rural. Sur un plan historique, il s'agissait pour moi de pallier les lacunes, en me centrant d'un point de vue archivistique sur les périodes de l'histoire coloniale qui n'avaient pas été étudiées. J'ai donc fouillé dans les archives du CHEAM créé en 1937 par Robert Montagne. C'était une institution de formation des administrateurs coloniaux et une boîte à idées pour concocter les politiques coloniales. J'y ai trouvé des rapports de stage d'une minutie extraordinaire, de même qu'à l'Ecole Nationale d'Administration. Par ailleurs, sur la période contemporaine, j'ai dû confectionner mes propres archives. J'ai collecté toute la presse $d u$ mouvement associatif, aussi bien en Kabylie qu'en France, depuisLienouTissuraf(publication du groupe d'études de Vincennes), jusqu'àTafsut(revue du MCB édité à Tizi-Ouzou), en passant par les feuilles volantes des associations de quartier. Un ami qui travaille à Air Algérie m'a longtemps rapporté périodiquement (et continue de le faire) 3-4 mois de plusieurs quotidiens algériens - à présent, on trouve tout cela sur le Net. J'ai même des " archives sonores » ainsi que des enregistrements de radio, par exemple au début du printemps 1980, quand Rachid Ali Yahia prétendait, sur les ondes de France Inter, être derrière la mobilisation citoyenne... J'ai également procédé à énormément d'entretiens, presque toujours de façon informelle, en prenant des notes, ou tout simplement à travers des conversations ordinaires. C'est depuis un an, à l'occasion des événements les plus récents, que je me suis mis à enregistrer plus régulièrement, pour garder une trace de cette histoire en cours. Mais à vrai dire, ma méthode dans les enquêtes menées de 1977 à 1991 n'avait rien d'académique, même si elle était systématique. En fait, c'était ma vie. J'étais plongé dans un milieu de Kabyles, en France, à Alger ou au bled, et je ne dissociais pas le recueil d'informations destinées à devenir des données, le bavardage à bâtons rompus ou la polémique intellectuelle et politique. J'ai engrangé, engrangé, engrangé, sans toujours savoir vers où je m'orientais.

Mais c'est peut-être moins l'accumulation de ces masses de papier imprimé que l'imprégnation de ce monde social où j'étais immergé depuis l'âge de 8-9 ans - quand 
j'ai commencé à fréquenter des foyers kabyles - puis la longue familiarité acquise au jour le jour, à travers les amitiés et les déceptions, les rencontres et les situations du quotidien, qui m'ont été utiles. D'abord, pour repérer les questions pertinentes, comprendre les trajectoires biographiques des uns et des autres, apprendre le sens des événements politiques tel qu'il apparaissait à mes interlocuteurs, me mettre à la place de mes enquêtés et voir les choses à travers leurs yeux. Ensuite, pour gérer les relations sur le terrain, savoir comment me comporter en situation - par exemple, je me présentais rarement comme chercheur ou doctorant, plutôt comme étudiant, «je m'intéressais », en évitant de prendre des notes sur le site... Je bénéficiais toujours de recommandations chaleureuses de la part d'amis, de parents d'amis etc., et n'étais presque jamais perçu comme un chercheur professionnel, plutôt dans la position du témoin et de l'invité.

Cette capacité de compréhension ou d'empathie ne saurait toutefois être fétichisée. On dispose de sources d'une grande richesse sur la Kabylie et les situations sont d'une extrême diversité. La stratification sociale y est très forte, allant du simple paysan au ministre. Le poids de la topographie locale y est très important, avec des différences dues aux contraintes naturelles du relief, aux types d'économie traditionnelle et aux formes d'investissement colonial. J'ai donc cartographié la région à partir de cartes dites d'état-major au $1 / 50000$, vivant pendant des mois avec ces cartes mises bout à bout sur un pan de mur de ma chambre. Ce qui m'a permis de dresser moi-même un fond de carte au 1/150000 - ce qui n'avait jamais été fait - en y projetant toutes les données trouvées dans les archives ou produites par les recherches de mes prédécesseurs: j'y ai placé les marchés, les écoles et leszaouias (couvents des confréries) ; dessiné les frontières des tribus et des confédérations, localisé précisément les plus gros foyers d'immigration.... Une carte de cette échelle n'existait pas - on a une carte Michelin au $1 / 300000$ qui n'était guère exploitable. Comme tu sais :la géographie, ça sert d'abord à faire la guerre... et pas plus en Algérie que dans le reste du Tiers-Monde on ne trouve de cartes à une échelle intéressante en vente sur le marché. J'ai trouvé ce que je cherchais à la cartothèque de la Bibliothèque Nationale. Ce fond de carte au 1/150000 a été capital. Il m'a permis de souligner l'hétérogénéité des situations à l'intérieur même de la Kabylie pour chaque période que j'ai traitée. J'ai pu souligner le poids des contraintes topographiques et voir comment celles-ci redoublaient souvent d'autres frontières, telles que celles de la distribution des écoles françaises ou des zones pourvoyeuses d'émigrés. Tout cela m'a permis d'identifier des sous-régions à la forte cohérence topographique et sociale.

Si l'on en vient au fond, comment as-tu redécouvert ce qui est devenu, au fil du temps, ton principal objet de recherche - les assemblées villageoises?

A partir d'un projet de thèse très ouvert qui était une anthropologie historique de la Kabylie sur deux siècles ${ }^{1}$, le fil rouge que j'ai tiré ou remonté, c'était la transformation des institutions municipales et de l'activité des assemblées villageoises en Kabylie depuis la conquête française. C'est la conjoncture politique qui m'a indiqué le chemin. En fait, j'ai suivi à peu près le même itinéraire que les militants issus du mouvement culturel berbère. Je m'explique. En désespérant d'établir à l'échelle régionale ou nationale l'espace de liberté auquel ils aspiraient, les militants du MCB se sont progressivement repliés sur la politique au village. Petit à petit, ils ont réinvesti latajmat(l'assemblée villageoise), se substituant à la génération des anciens dans la gestion des affaires locales. Les problèmes de construction et d'entretien des routes, des fontaines et des adductions d'eau constituent la part la plus importante de l'activité destajmat, qui pallient ainsi le déficit des services publics de l'Etat en la matière, pour cause d'inefficacité, de corruption ou 
de bureaucratie, toutes les plaies du Tiers-Monde. C'est-à-dire qu'en s'impliquant activement dans les affaires civiques au sens strict, les jeunes issus du MCB ont réimpulsé l'activité des assemblées villageoises en suscitant, par exemple, la mise en place de Maisons de jeunes ou d'associations culturelles. Entre 1984 et 1987, il y a eu un rajeunissement systématique de l'âge moyen des membres des comités de village, qui passait de $60 / 70$ ans environ à 40/50 ans. Ma chance est d'avoir fait mon travail de terrain juste à ce moment-là et d'avoir trouvé des interlocuteurs plus jeunes et davantage sensibles à mon projet de connaissance que la génération des anciens.

Pour autant, cette situation ne me dispensait pas de la nécessité d'obtenir toutes sortes de recommandations pour parvenir aux lieux d'enquête et avoir connaissance des affaires intimes des villages. La tenue des réunions des assemblées villageoises requérant le respect de règles de confidentialité. Entre 1985 et 1987, dans trois villages, j'ai quand même réussi à faire des clichés des procès-verbaux des réunions, en dissimulant les noms de personnes et de lieux qui figuraient sur les registres. Reste, évidemment qu'il ne m'était pas possible de participer directement à des réunions : qui n'est pas citoyen du village ne peut y participer. Une des règles fondamentales qui régit le fonctionnement de ces assemblées est précisément de sanctionner celui qui aurait divulgué à l'extérieur les affaires qui y sont traitées. En revanche, dans deux cas, le comité du village, qui est une sorte de conseil permanent qui intervient comme cellule de crise, s'est concerté et a accepté de se réunirad hocpour discuter avec moi de tous les problèmes qui m'intéressaient. La condition mise à chaque fois, était de respecter la clause de confidentialité.

Pour moi, ce travail d'enquête sur le terrain a été d'emblée connecté avec un travail de réflexion sur les théories disponibles et de relecture des textes classiques. Pour aller vite, en anthropologie du Maghreb, la théorie de la segmentarité revisitée par Ernest Gellner - et déclinée brillamment sur la Kabylie par Jeanne Favret - organise le champ des questions pertinentes en anthropologie depuis les années 1970. La logique du jeu segmentaire est fondée sur l'articulation de liens généalogiques, réels ou imaginaires. J. Favret a souligné l'aporie que représentent les assemblées villageoises et s'est heurtée à l'impossibilité de penser le lien entre territorialité et généalogie qui caractérise le village. Ce qui dans l'expérience vécue m'apparaissait incontournable, cette forme d'activité de la vie du village, était du coup effacé par la théorie. L'autre grand modèle, c'est Bourdieu. Alors, évidemment, il y a le Bourdieu duDéracinement,Algérie 60,Travail et travailleurs, qui est une sociologie bétonnée avec enquêtes empiriques, encore que le contexte et les modalités de la collecte des données n'apparaissent pas toujours - loin de là-, et il y a le Bourdieu de l'Esquisse d'une théorie de la pratiqueet duSens pratique, qui nous présente une Kabylie éternelle, sans rapport avec la Kabylie contemporaine, fondée sur des enquêtes menées avec Abdelmalek Sayad et sur les documents disponibles. Dans ces deux livres, sa perspective est celle d'une économie générale des pratiques, de la dialectique du champ et de l'habitus et de l'orchestration sans chef d'orchestre d'une "logique pratique» ou d'une "grammaire invisible ». Là, pas de place pour les médiations: la présentation des institutions villageoises relèverait d'unjuridisme d'ethnologue. Et d'ailleurs, Bourdieu dans ses travaux rabat l'assemblée villageoise - sans la nommer vraiment - au rang du conseil de famille informel, simple projection de l'ordre lignager, qui ne donne pas à penser. Le défi pour moi était de forger les outils descriptifs et analytiques pour comprendre la vie politique et juridique occultée par les deux modèles. Latajmatm'est peut-être apparue avec toute son importance parce que je n'avais initialement pas de théorie toute faite et parce que j'avais du coup une forme de disponibilité à ce phénomène; d'autre part, parce que ma lecture devait plus à l'ébranlement par les 
événements des années 1980 qu'à la poursuite d'une thèse académique. En fait, je me suis confronté théoriquement à l'assemblée villageoise un peu comme les jeunes militants issus des rangs $d u M C B$ se sont mis à réinvestir les affaires du village! Je l'ai redécouverte comme les militants $d u M C B$, en leur compagnie du reste, avant d'en refaire un objet pertinent pour les sciences sociales. C'est l'aventure d'une génération d'hommes du même âge que moi et c'est la puissance propre à un mouvement social qui ont aiguillé mon attention théorique vers ce phénomène.

Du même coup, la littérature qui en avait parlé pendant près d'un siècle, mais avait connu un coup d'arrêt dans les années 1950 avec la montée du mouvement nationaliste, puis avait été refoulée par l'exigence d'unification socialiste de la nation algérienne, a retrouvé pour moi tout son intérêt. Dans l'espace intellectuel, cette littérature avait perdu de son attrait et de son crédit à la faveur du déclin de l'anthropologie juridique, de la prédominance de la vision structuraliste et de la critique de la complicité entre ethnologie et colonialisme. Le dernier à en parler avait été Bousquet. Entre 1950 et 2000 , rien.

Pourquoi en avait-on autant parlé au tournant du XIXe au XXe siècle ? Et pourquoi ce blanc à partir des années 1950 ?

Divers types d'administration locale mis en place par le pouvoir colonial en Kabylie avaient commandité et favorisé toutes sortes d'enquêtes. L'ethnographie fondamentale de Hanoteau et Letourneux, dansLa Kabylie et les coutumes kabyles ${ }^{2}$,avait pour but explicite de servir à l'administration judiciaire de la région. Alors évidemment, dans cette énorme production éditoriale, il y a eu le meilleur et le pire. On dispose ainsi d'une ethnographie très minutieuse, qui a surtout été produite par des officiers, des administrateurs, des magistrats, etc. mais très peu d'universitaires.La formation des cités deMasqueray ${ }^{3}$ est une exception. Mais on trouve aussi n'importe quoi dans cette littérature, toutes sortes de délires qui ont fini par nourrir la vision de l'anthropologie comme fille de l'impérialisme. La Kabylie de ce point de vue-là était complètement disqualifiée, scientifiquement et politiquement - alors même qu'elle avait étayé d'éminentes hypothèses théoriques, depuis Renan, Tocqueville ou Durkheim. Une partie de mon travail est aujourd'hui d'exhumer cette masse de références à la Kabylie, de montrer à la fois ce qui doit en être retenu et ce qui doit être abandonné. Bref, une sorte d'histoire de l'anthropologie vue de Kabylie.

Il y a donc un lien étroit entre ton engagement intellectuel, extrêmement fort, tes attaches biographiques avec la Kabylie, tes positions morales et civiques et ta réceptivité aux événements politiques. Quel sens accordes-tu à ton travail dans cet horizon?

Un mot d'abord sur l'écriture de ma thèse, qui soulève la question de l'engagement et du retrait. Il a fallu que j'émigre pendant près de cinq ans en Orient pour écrire ma thèse. Mon épouse était pensionnaire à l'Institut d'études arabes à Damas. Un exil doré, mais une situation d'émigration. Après quelques tentatives d'enquête sur un quartier de Damas où il y a eu traditionnellement une émigration kabyle, je me suis retranché dans mes archives. Sans cette phase d'isolement presque total, je ne serais pas parvenu à penser mon objet et à auto-analyser mon rapport à lui. Mon seul lien avec la Kabylie restait l'abonnement au journal local de Tizi Ouzou: Thamurth. L'Algérie traversait à l'époque cette phase de violence extrême, où pour ma part, je ne comprenais plus rien. Qui tuait qui? Le chaos. Rares sont ceux qui, en 1992-94, comme Mohammed Harbi[ancien membre du FLN, conseiller de Ben Bella à partir de 1962, emprisonné par Boumédiène de 1965 à 1973, aujourd'hui historien de l'Algérie], entrevoyaient la collusion entre Etat et terrorisme. Le mouvement culturel berbère était mort et 
enterré, rien d'autre ne se profilait à l'horizon. Impossible de retourner en Algérie, où je suis allé la dernière fois en 1992 - la crainte d'exposer au danger les personnes qui me recevaient et m'hébergeaient à l'époque. Il ne me restait qu'à prendre la fuite dans l'histoire et la théorie. Le lien est presque direct entre les événements politiques, qui enjoignent à poser de nouvelles questions, et mon activité de chercheur. Depuis un an, par exemple, la sommation de l'actualité me pousse à écrire un petit livre pour en rendre compte - et j'ai laissé toute autre affaire en suspens. Cet éloignement a aussi eu une autre vertu. Il m'a mis dans une situation de comparaisonin vivoentre Maghreb et Machrek. La compréhension vécue de la culture politique, la perception des traces de l'histoire coloniale, si courte en Syrie... J'ai pu voir l'équivalent de ce que l'Algérie avait perdu - une qualité de la vie, des modes de consommation, des activités artisanales et artistiques... J'ai pu mesurer aussi à quel point je partage avec les Kabyles un sens de l'honneur, de l'inégalité et de l'injustice, qui se traduit par des formes de résistance ou de révolte, bref, une sorte de passion pour la chose publique - ce que certains régimes politiques, avec leur quadrillage policier de la population et leur pratique de l'arbitraire et de la terreur, ont réussi à étouffer.

Quand je suis revenu en France, j'ai réactivé mes réseaux de relations, retrouvé de vieux amis. Je suis reparti un an en Tunisie, puis entré à l'Ecole des Hautes Etudes. J'y suis secrétaire d'une association,Barbaresques, dont l'activité, en sommeil par manque de ressources, est d'inviter en France des chercheurs maghrébins hors réseaux.Par ailleurs, mon séminaire, Anthropologie juridique et politique des sociétés arabo-berbères, a attiré pas mal de Kabyles, à tel point que j'ai décidé de lancer un séminaire, fermé, où l'on ne traite que de la Kabylie contemporaine. Cette position institutionnelle a donné davantage de visibilité à mon travail et a renforcé les liens que j'entretenais avec des chercheurs kabyles. Par ailleurs, j'ai eu la chance de rencontrer Abderahmane Bouchene, pratiquement le premier éditeur privé qui est apparu dans les années 1980 en Algérie où il est devenu, disons, indésirable. A tel point qu'il en est parti de façon précipitée. On s'est très bien entendu, avant tout politiquement... Je me suis retrouvé engagé, pratiquement à mi-temps, dans la vie de cette maison d'édition, où je m'occupe de la direction d'une collection, et où nous travaillons aussi bien à la réédition d'ouvrages classiques, épuisés de longue date, sur le Maghreb, et où nous prospectons des manuscrits inédits. Le projet de Bouchene part du constat de la méconnaissance du Maghreb contemporain, en particulier de l'Algérie, sur laquelle on n'a presque rien publié depuis une trentaine d'années en dehors de recherches sur l'islamisme dont le moins que l'on puisse dire est qu'elles sont inégales... Pendant longtemps les thèses consacrées à l'Algérie ont porté sur des politiques publiques volontaristes sans rapport avec le pays réel. C'est ainsi qu'on trouve un nombre incroyable de recherches qui ont scruté l'avènement du socialisme en Algérie. Totalement caricatural! Ce déficit de connaissance et cette opacité continuent d'avoir des effets dans le débat public. Avec Bouchene, nous concevons notre tâche comme une contribution à la vie civique et politique des pays où nous éditons et diffusons nos livres.

A vrai dire, depuis vingt ans, je ne me suis jamais senti aussi engagé que maintenant. Au vu des derniers résultats électoraux en France, et de la percée de l'extrême droite, je me rends compte à quel point ma vocation de citoyen est tournée vers la Kabylie. Je suis français et n'ai jamais eu aucun doute là-dessus. Mis à part, peut-être à l'adolescence, quand toutes les identités se mêlent et que, dans un état de confusion, on est prêt à endosser une autre culture. Mais aujourd'hui, surtout depuis le printemps noir de 2001, "depuis que la Kabylie réinvente la Commune" - le titre d'un papier écrit récemment pour Alternatives internationales - c'est là que mon engagement se joue. Depuis que j'ai publié ma thèse, où les deux derniers chapitres traitent de l'actualité de 1994 à 2000, et que j'ai entrepris 
d'interviewer les représentants de la direction tournante des comités de villages et de communes de Kabylie, je me sens pleinement, plus que jamais, engagé en faveur de cette cause.

Mais quel type de difficultés as-tu rencontré à passer de la relative tranquillité du dépouillement d'archives, quand tu t'inscris dans une perspective d'anthropologie historique, au bruit et à la fureur de l'actualité, quand tu tentes de discerner le sens des événements sur le vif?

Pour couvrir les événements des années 1980, j'avais déjà eu de nombreux tiraillements, éthiques, politiques, déontologiques. Est-ce que je dis tout? Jusqu'où je peux aller? Qu'est-ce qu'il vaut mieux passer sous silence? Quelles seront les conséquences de ce que je rends public? Dans quelle mesure je risque de donner des arguments au pouvoir ou à ses relais pour discréditer un mouvement citoyen démocratique (le MCB)? En montrant les zones d'ombre du discours identitaire et en dévoilant certains de ses enjeux stratégiques, le risque était réel d'affaiblir le mouvement et de faire le jeu de ses adversaires. Finalement, j'ai presque tout dit. En revanche, quand il a fallu réfléchir sur l'actualité immédiate, depuis 1994, il me manquait beaucoup d'informations. Les intrigues en coulisses et les conflits de leadership ne m'avaient pas directement intéressé pour la rédaction de la thèse. Mais ce qui était rejeté dans la petite histoire, sans pertinence pour mon propos anthropologique, a pris une saillance inattendue quand j'ai fait des entretiens avec des animateurs de premier plan du MCB qui se sont exilés à Paris - je peux citer en particulier le nom de Ahssan Taleb et de Ramdane Achab, ainsi que quelques autres que je ne remercierai jamais assez pour les longues séances de travail que nous avons eues ensemble. Les problèmes se sont tout de suite imposés. Comment rendre compte d'une situation qui n'est connue que par deux ou trois témoins et sur laquelle aucune preuve ne peut être avancée? Sur quels critères se fonder pour en parler ou ne pas en parler? C'est tout le problème de l'histoire en train de se faire, de distinguer entre ce qui vaut comme archive et ce qui est pure conjecture. De quelle façon évoquer une réunion de leaders d'organisation où des décisions importantes sont prises, mais destinée à rester dans l'ombre des tractations politiques? Quand le travail d'infirmation ou de confirmation des preuves ne vaut plus, et quand le recoupement des indices n'est plus pleinement satisfaisant, le seul critère de vraisemblance et de pertinence qui nous reste est celui de l'analyse politique. Les descriptions et les analyses que l'on avance alors ne sont pas de l'ordre du fait avéré, mais de l'ordre des présomptions, elles présentent des faits plausibles qui s'inscrivent comme des épisodes dans une intrigue, étayée par toutes sortes d'indices. Que ce soit dans une affaire politique ou judiciaire, on est toujours renvoyé à ce critère ultime. Dans un grand nombre de cas, on ne dispose ni de procès-verbal, ni de traces d'archives, a fortiori quand il s'agit d'actions secrètes ou de réunions clandestines. Quant aux récits après coup, ils sont ré-élaborés par le travail de la mémoire ou carrément réécrits à des fins stratégiques. Si l'on prend l'affaire Matoub Lounès, par exemple, l'un des chanteurs kabyles les plus populaires, devenu l'un des leaders les plus véhéments du mouvement culturel, son enlèvement par un supposé groupe d'islamistes en septembre 1994 avait mis la Kabylie en ébullition. Elle s'acheva par sa libération dans des conditions mystérieuses. Pourquoi les "islamistes" n'avaient-ils pas exécuté l'un de leurs pires ennemis? Certains se mirent à suspecter le RCD d'avoir profité de cet enlèvement, qui lui avait assuré un regain de recrutement et lui avait permis de multiplier les manifestations de popularité pendant l'enlèvement de Lounès lui permettant ainsi de se placer à la tête de la constitution de milices de patriotes. D'autres firent le rapprochement avec les négociations engagées entre le clan du Président Zeroual et du général Betchine avec le leader du FIS, Abassi Madani, et l'accord du FFS d'Aït Ahmed, en vue d'une solution politique: le coup pouvait avoir été monté par un clan rival opposé à cette solution politique. Selon la fondation Matoub Lounès, créée le lendemain de sa mort, le chanteur, d'abord dupe, se mit à avoir des doutes sur l'identité de ses ravisseurs et s'apprêtait à le déclarer 
publiquement. Beaucoup estiment aujourd'hui, version plausible, qu'il a été exécuté pour cette raison avec la complicité du Rassemblement pour la culture et la démocratie (RCD), à la solde d'un clan du pouvoir. Même si aucune donnée vérifiable n'est disponible pour attester de cette lecture des faits, c'est aujourd'hui la conviction du plus grand nombre de citoyens et d'observateurs du mouvement culturel berbère.

Ce travail d'établissement des faits et d'élaboration de présomptions a été extrêmement pénible, traversé de disputes très vives avec mes interlocuteurs, de séances où l'on s'est fâchés, de ruptures de deux mois après deux mois de collaboration. Tout reposant sur l'analyse politique, les dissensions avec mes principaux informateurs, - pour certains des leaders du mouvement - signifiaient des désaccords sur le sens des faits qu'ils me révélaient. L'un d'entre eux, en tant qu'acteur immédiat, avait des visées stratégiques dans la transmission de ces informations, et avait ses propres raisons le conduisant à être, selon moi, aveugle sur certains points; de mon côté, j'étais intéressé par les faits qu'il me racontait, mais que je mettais en perspective par rapport à d'autres conjectures politiques ou phénomènes socio-politiques. C'était des tiraillements incessants, tant dans les discussions que dans la relecture des textes que je lui soumettais, où il me demandait de gommer tel nom, où il contactait les personnes citées pour s'assurer de leur accord à être mentionnées, et où souvent, il formulait son pur et simple refus de mes analyses politiques. Nous avions commencé à écrire à quatre mains, ce qui s'était vite avéré impossible. On passait des soirées entières à relire chaque page, à réécrire chaque version, à la réévaluer et à la rectifier un processus qui a été au bout du compte fructueux, mais où il a fallu donner beaucoup de temps et d'énergie et que nous avons, lui et moi, vécu dans la tension...

Vérification des données, si possible, constitution de faisceaux de présomptions, échanges avec des informateurs privilégiés pour établir des faits et éprouver des intrigues... Est-ce que tu penses que l'anticipation de la réception de ton texte par certaines agences de l'Etat algérien ou français ou par des acteurs sociaux ou politiques a eu des effets sur ton travail d'écriture ? Et comment le travail effectif de réception de la part des lecteurs a-t-il rejailli sur ta recherche après publication?

Par rapport à l'Etat algérien, vu le sujet et ma posture, je me souciais comme d'une guigne de son avis. De toute façon, de nos jours ils accordent rarement le visa aux chercheurs qui veulent faire $d u$ «terrain » en Algérie. Rien à y gagner et rien à y perdre. Pour les acteurs, je savais ce que je voulais. Je voulais contribuer à établir des faits, les mettre en perspective par rapport au reste de ma recherche, et surtout, participer à affiner les analyses qui s'élaboraient au sein du mouvement citoyen. Dans certains cas, il ne me déplaisait pas de chercher à avoir raison contre tous. Par la suite, au grès des développements politiques de ses dix dernières années, il s'agissait avant tout d'établir des faits et de promouvoir une analyse politique contre la langue de bois des deux principales formations politiques qui se partageaient les scrutins des Kabyles - le FFS et le RCD. Paradoxalement, dans le milieu universitaire kabyle, je suis apparu très tôt, à travers la circulation de ma thèse à partir de 1995 - avant la publication du livre en 2001 - comme un proFFS. Même si je l'égratignais pas mal, et s'il m'a démontré par la suite que mes analyses le dérangeaient, j'étais catalogué comme tel, au vu également de mon réseau de relations. Aujourd'hui le FFS me parait totalement à côté de la plaque, soupçonnant systématiquement la moindre initiative politique faite en dehors de lui d'être le produit de manipulation des "services ». Bien qu'on ait entendu des déclarations contradictoires, c'est encore la position courante des leaders FFS à l'égard du mouvement citoyen contemporain. Comment soutenir sérieusement que plus d'un an de mobilisation quasi permanente d'une région entière est le 
résultat d'une manipulation du pouvoir, et à quelle fin? En fait, le mouvement citoyen a marginalisé toutes les vieilles structures politiques et celles-ci ont perdu les repères de jugement pour comprendre la situation. Dès que quelque chose leur échappe, ils avancent la thèse paranoïaque de la machination d'Etat. La mobilisation citoyenne reste pour eux une créature des services secrets.

La forme de crédit dont je bénéficie actuellement provient du fait que, dès avant l'avènement du mouvement citoyen - mon bouquin est sorti exactement trois mois avant - dans la conclusion de mon travail, j'avais identifié les assemblées de villages comme le lieu où se concentraient et convergeaient les ressources politiques, les réseaux militants et les structures organisationnelles susceptibles d'ancrer une résistance organisée au pouvoir. Cela a facilité la prise de contact auprès de quelques représentants des coordinations du mouvement citoyen, venus à Paris la plupart pour échapper à la répression. Depuis fin mars 2002, l'Etat a entrepris d'arrêter les leaders de cette coordination, pourtant tournante, usant de toutes les tactiques possibles et imaginables pour discréditer un tel, laisser croire que tel autre a été retourné, faire circuler des rumeurs sur un troisième, emprisonner de façon sélective les leaders, et ainsi de suite. Pour ma part, la publication du livre a été bien reçue par nombre de militants, y compris du FFS - et relativement bien diffusée, la première édition à 1200 exemplaires a été épuisée au bout d'un an. Au plus fort des manifestations, en juin 2001, mon passage à la télé sur LCI pendant 6-7 minutes m'a aussi servi, et dans plusieurs manifestations parisiennes auxquelles j'ai participé, $j^{\prime} a i$ noté que certains m'avaient reconnu. Petit à petit, la réputation fait que l'on a accès à de nouveaux matériaux et que l'on peut rencontrer de nouveaux interlocuteurs, accroitre ses réseaux de relations, en partant du cercle de la famille, des familiers des familiers, des amis de longue date, des cercles de militants, puis des liens noués à la faveur des publications. De fil en aiguille, on barre des noms dans son carnet d'adresses, des gens qui s'avèrent peu fiables ou qui ont mal tourné, on en inscrit de nouveau. Le travail de l'information et de l'analyse est indissociable de cette dynamique de rencontres. L'approfondissement de la compréhension des événements en dépend. Et la réception de mes prises de position vaut comme une série d'épreuves à travers lesquelles je gagne ou perds du respect et peux continuer mon travail de façon pertinente ou pas. L'affectif et le politique sont là aussi déterminants. Si j'ai pu pénétrer dans les villages, c'est parce que les gens ont vu dès le départ que c'était pour moi un investissement lourd à fonds perdu, que j'étais mu par la passion et non par quelque intérêt. Qui se ressemble s'assemble.

Dans la plupart des manuels sur l'enquête, on évoque le type de rétributions matérielles et symboliques qui servent à payer les « indigènes ». Comment interprètestu ton rapport à des « informateurs»?

Il faut donner du temps et de soi. J'ai passé des centaines d'heures à parler de l'actualité et de l'histoire de la Kabylie. A Boghni, par exemple, j'ai retrouvé les démêlés au début du XIXe entre deux arch (tribus), Ait Mendes et Ait Koufi, que les habitants ignoraient, alors qu'ils sont toujours en conflit. Je compensais l'inégalité des rapports avec ces discussions dont mes interlocuteurs sont avides. Une chose importante, c'est l'asymétrie des relations que j'ai vécues sur le terrain. Je n'ai jamais été en train de calculer les rétributions des informations qu'on me donnait, comme on le veut d'ordinaire du travail de l'ethnologue, qui doit apporter des cadeaux et de la nourriture, rendre des services et être utile d'une façon ou d'une autre. Moi, on m'a toujours reçu, accueilli avec hospitalité, et je mettais un certain temps à donner des cadeaux en retour, pour éviter que ce soit perçu comme un paiement déguisé. Mon amitié, ma connaissance et ma disponibilité suffisaient. Ça n'est pas allé sans mésaventures, comme dans toute histoire, et j'ai beaucoup réfléchi, avec toi d'ailleurs, aux vicissitudes de l'échange de dons. Et j'étais vigilant 
à mettre en place des relations asymétriques, évitant même que mon travail apparaisse au bout du compte comme un retour d'investissement de la part de mes informateurs. L'amitié entre personnes et la reconnaissance d'une analyse politique ne sont pas monnayables. Quand j'ai envoyé mon livre aux cinq comités de village qui m'avaient ouvert leurs portes, je l'ai fait comme un acte de civisme, et comme un acte de remerciement et de reconnaissance envers les personnes qui m'avaient informé. J'ai fait porter mon livre, faute de pouvoir le porter moi-même. Il y a eu quelques comptes-rendus dans la presse algérienne, que je n'ai jamais eus sous les yeux. Mais ce qui joue le plus, c'est le bouche à oreille.

Et ton prochain livre, ce récit des événements de l'année 2001-2002, où tu rassembles des témoignages et des documents, à quoi et à qui le destines-tu ? La démarche est-elle très différente de la publication de ta thèse, et en quoi ? Tu te vois comme un témoin, un archiviste, un journaliste, un expert?

Ce livre, c'est tout autre chose. Un livre à chaud sur l'histoire en train de se faire, qui produit les archives du mouvement - les procès-verbaux des réunions notamment-, qui établit des faits et en invalide d'autres grâce à une analyse politique serrée. Pour cela, en dehors des sources documentaires, je m'appuie sur des entretiens avec des membres des coordinations $d u$ mouvement citoyen. Ce que je veux faire avant tout, c'est à la fois écrire une histoire en train de se faire et, en même temps, inscrire cette mobilisation citoyenne dans le contexte socio-politique de l'Algérie contemporaine, dans les traditions municipales et la culture politique propres à la Kabylie. Raconter cette série de 22 ou 23 " conclaves " qui ont déjà eu lieu. Le terme, emprunté au vocabulaire ecclésiastique et dénotant d'ordinaire les réunions de cardinaux, désigne ici les assemblées des coordinations de comités. Peut-être a t-il été choisi par un journaliste et s'est-il imposé ensuite au mouvement parce qu'il permet de rehausser la solennité ou la sacralité de l'espace public qu'ils instituent, en écho à ce que j'ai appelé dans ma thèse le "sacré laïc ", la horma du village? Mais il faut être prudent avec ces rapprochements. On est maintenant dans des assemblées aux procédures, au mécanisme de représentation par mandat, aux ordres du jour, et ainsi de suite, empruntés à la culture politique moderne. On assiste également à une évolution des processus de prise de décision: au début de la CADC (Coordination des Archs, Daïra et Commune, de Tizi Ouzou), les décisions étaient prises à l'unanimité, à l'image du mode de fonctionnement qui prévalait dans les assemblées de villages. Depuis juillet/août 2001, les coordinations sont en train de redécouvrir un principe démocratique - pas encore un principe de majorité, pour l'instant un principe des trois quarts. Tout un processus de mandats et de consultations a été mis en place. Certaines procédures plus anciennes fonctionnent encore. On en retrouve des traces dans lecode d'honneurdont s'est doté le mouvement. Plus récemment encore, on parle d'ostraciser les délégués des coordinations qui enfreindraient la décision de boycotter les élections législatives - du 30 mai 2002 - en briguant un mandat. On a assisté à des cas de menace et de début de lynchage, avec les "délégués[made in]Taïwan" notamment, qui avaient usurpé leur mandat à la table de négociations. Mais petit à petit, c'est une capacité à gérer une minorité d'opposants, à reconnaitre la division et le conflit et à rendre viables la prise de décisions qui mûrit. Dans ces assemblées des coordinations, on retrouve des dizaines de délégués représentants des centaines de milliers de Kabyles.

Ce travail d'information et d'analyse, je veux l'inscrire également dans la longue durée, le recadrer par rapport à un héritage. Il me semble, par exemple, capital de lever une série de malentendus autour du terme de "tribu», utilisé de façon indue par les médias, qui le lient à l'imagerie archaïque de la tribu biblique, qu'on évoque aussi à propos du Yémen ou de l'Afghanistan. En Kabylie, une réunion de tribus procède davantage d'un conseil d'administration. Ce processus de modernisation politique est 
également en cours à l'échelle des villages, et cela depuis le début du siècle. J'ai déjà évoqué le passage au français dans la transcription des procès-verbaux des réunions des assemblées villageoises sur des cahiers d'écolier ou des registres de commerce. Avant, leqanun (droit coutumier)du village, quand il était écrit, l'était en arabe, langue sacrée, et paraphé par les marabouts.

Les communes actuelles de Kabylie (issues du découpage de 1984), coïncident dans beaucoup de cas avec le tracé des frontières tribales, tel que je l'ai reconnu d'après les archives datant de la première moitié du XIXe siècle. Qu'est-ce à dire? Que les tribus se sont pérennisées! Oui et non! En fait, pour faire simple, on pourrait dire que le mot et l'espace de la tribu ont connu une substitution quasi-totale de leur contenu. Les tribus ont cessé d'être des unités politiques et guerrières depuis la conquête coloniale. En revanche, compte tenu du fait qu'une tribu correspondait le plus souvent à une entité topographique - un versant de montagne, un pâté de colline -, les gens qui l'habitent ont continué à s'identifier comme membres d'un même ensemble. D'autant que la modernisation du pays, grâce à l'infrastructure routière et aux équipements collectifs, a renforcé l'intégration de ces unités topographiques. Les gens d'une commune/tribu fréquentent les mêmes marchés, services publics, polycliniques, gendarmerie. Autant d'équipements collectifs, d'espaces publics et de réseaux de sociabilité qui renforcent le sentiment d'appartenance à un même ensemble. Ce à quoi je me suis attaché, c'est de mettre en évidence la continuité d'une culture politique plus "traditionnelle », avec toutes sortes d'innovations qui font surgir un nouveau type de collectivité politique. Et l'une de mes visées, c'est de travailler à la réconciliation des Kabyles avec leur histoire, que le pouvoir s'est acharné à discréditer. Les arouch, ce n'est pas de l'archaïsme comme la critique de la tradition le prétend, ce n'est pas non plus simplement une ressource qu'on utilise pour des raisons stratégiques. C'est un héritage tout à fait moderne: les unités topographiques et toponymiques ne renvoient plus à des collectifs belliqueux et des entités mythiques, comme autrefois, mais elles restent des unités d'aménagement du territoire et d'échange commercial. La loi de 1984 de re-découpage des communes n'a fait rien d'autre que reproduire par pur pragmatisme l'espace des tribus, en se fixant sur les réseaux routiers ou les équipements collectifs.

Pour revenir à la situation qui prévaut depuis plus d'un an, il faut dire qu'on est dans une situation insurrectionnelle en Kabylie : les gendarmeries ont été assiégées et, pour un bon nombre, évacuées et démantelées (c'était une des principales revendications de la plate-forme du mouvement citoyen, dite d'El Kseur). En un an, la répression a fait plus de cent morts, la mobilisation faite de sit-ins, de manifestations et d'émeutes spontanées est presque permanente. Toutes les semaines à l'échelle communale et tous les quinze jours à l'échelle régionale, on a des assemblées politiques. Mais la mobilisation déborde de beaucoup ces lieux de pouvoir. Les assemblées légifèrent sur leur mode de fonctionnement et de représentation interne, choisissant une direction tournante et un site tournant de façon à éviter une cristallisation des positions de pouvoir et une infiltration par les services secrets. Elles légifèrent aussi à l'extérieur : pas pour tracer un nouveau territoire et faire sécession de la nation algérienne, ni pour établir une nouvelle constitution et affirmer la souveraineté d'un nouvel organe politique, mais pour tenter de faire tomber le régime des généraux. Au départ, les revendications étaient assez minimalistes, dans un cadre terrible : 150000 morts après dix ans de troubles, l'économie exsangue. Un des slogans des jeunes était «vous ne pouvez pas nous tuer, nous sommes déjà morts». Puis, un jeune de vingt ans, Massinissa Guermah se fait assassiner dans les locaux d'une brigade de gendarmerie à 
la veille de la commémoration du printemps berbère de 1980, le 20 avril... une provocation destinée à mettre le feu aux poudres. C'est dans la bourgade même de l'assassinat (Beni Douala) que l'idée d'un comité a germé pour demander le départ des brigades de gendarmerie recrutées à travers toute l'Algérie et montées contre les Kabyles. L'idée d'une coordination a ensuite fait boule de neige dans toute la région. Une hiérarchie d'emboîtements s'est mise en place : comités de village et de quartier pour Tizi Ouzou et Bejaia, comités de communes, comités de daira (circonscription administrative) et comités interdépartementaux, qui associent une dizaine de départements $d u$ reste de l'Algérie. Aujourd'hui, nous en sommes à la veille d'un boycottage massif des élections législatives, avec une prise de position de nombre de maires contre le scrutin : on s'achemine vers une participation électorale dérisoire. Seules les forces de sécurité et les fonctionnaires locaux issus d'autres régions risquent d'aller voter. L'objectif est donc de faire chuter le gouvernement actuel. Il est aussi d'échapper à la conception culturaliste qui a été celle du mouvement citoyen des années 1980. Pour moi, le nouveau danger à éviter est celui de l'autonomie agitée par des leaders politiques qui traînent pourtant plus d'une casserole... Jusqu'à présent le mouvement citoyen a été très prudent sur ce point, mais saura-t-il contenir les plus jeunes et les plus désespérés? La montée de la haine entre Arabes et Kabyles, entretenue par le pouvoir depuis l'indépendance et exacerbée par les dix dernières années de terreur, fait le lit des projets politiques dont les promoteurs risquent d'être les seuls à recueillir les fruits. Le discours antiarabe fonctionne: "Ce que le bouf laboure, l'âne le mange». Combien de jeunes Kabyles ne s'identifient-ils pas spontanément au bouf du proverbe? L'enjeu politique, que je vise aussi à travers la préparation de mon livre, est de soutenir la perspective nationaliste et démocratique du mouvement, et éviter toutes les dérives qui font selon moi le jeu du pouvoir.

Si tu devais caractériser ton travail, tu le verrais donc comme celui d'un anthropologue, sociologue ou historien? D'un militant, d'un avocat ou d'un expert?

Anthropologue, sociologue ou historien, pour moi c'est la même chose. Anthropologue citoyen, c'est ce qui m'irait le mieux. Expert, pour les Renseignements généraux ou les Affaires étrangères, je refuse de l'être. La seule fois où j'ai fait le "conseiller du prince", c'était dans le cadre d'un rapport pour la Direction des populations migrantes (DPM), je l'ai fait en évitant toute information de type recensement ou policier. J'ai parlé de la mobilisation politique dans l'immigration sans mouiller personne et de façon inexploitable pour un service de police. Malgré quelques réticences, la direction de la DPM était plutôt contente. Pour la Kabylie, je me suis demandé: s'ils viennent me voir, qu'est-ce que je ferai ? Mais en fait, étant donné les relations entre la classe politique française et les généraux d'Alger (sans parler de la collaboration entre les services secrets entre les deux pays, comme on a pu le voir dans l'affaire Mecili), je doute qu'un tel rapport soit utile. La seule attente à laquelle je veuille répondre, c'est celle des militants et des citoyens kabyles, et mon défi, quelque peu narcissique, c'est d'avoir raison contre tout le monde. De réhabiliter la culture politique kabyle qui n'a plus grand chose de "traditionnel» contre le soupçon d'archaïsme qui pèse sur elle, et de montrer son importance dans la mobilisation actuelle. Anthropologue citoyen, ça me convient. 


\section{NOTES}

1. . Mahé A., Histoire de la Grande Kabylie XIXe-XXe siècles. Anthropologie historique du lien social dans les communautés villageoises, Saint-Denis, Bouchène, 2001.

2. . Hanoteau A., Letourneux A., La Kabylie et les coutumes kabyles, Paris, A. Challamel, 1903.

3. . Masqueray E., La formation des cités chez les populations sédentaires de l'Algérie. Kabyles du Djurdjura, Chaiuïa de l'Aourâs, Beni Mzâb, Paris, E. Leroux, 1886.

\section{RÉSUMÉS}

Alain Mahé has been travelling Kabylie, its history and rites for more than twenty years. In this interview he remembers his experiences and the way they supported his work of enquiry, and how he considers them inthe light of his personal researcher trajectory. In this interview, Alain Mahé recalls how his career and research topics are linked to his personnal trajectory, his friendships and childhood in a suburb south Paris.

\section{INDEX}

Index géographique : Maghreb

Mots-clés : anthropologie 